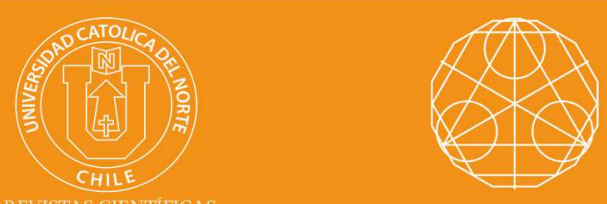

\title{
On asymptotic commutativity degree of finite groups
}

Rajat Kanti Nath* iD orcid.org/0000-0003-4066-5608

"Tezpur University, Dept. of Mathematical Sciences, Sonitpur, AS, India.

rajatkantinath@yahoo.com

\section{Abstract:}

The aim of this paper is to give a detailed account of a problema posed by $P$. Lescot regarding asymptotic commutativity degree of finite groups.

Keywords: Finite groups; Commutativity degree.

MSC (2010): 20D40, 20D60, $20 \mathrm{P} 05$.

\section{Cite this article as (IEEE citation style):}

R. Nath, "On asymptotic commutativity degree of finite groups", Proyecciones (Antofagasta, On line), vol. 38, no. 4, pp. 829-835, Oct. 2019, doi: 10.22199/issn.0717-6279-201904-0054. [Accessed dd-mm-yyyy].

Article copyright: (c) 2019 Rajat Kanti Nath. This is an open access article distributed under the terms of the Creative Commons Licence, which permits unrestricted use and distribution provided the original author and source are credited. 


\section{Introduction}

The commutativity degree $d(G)$ of a finite group $G$ is the probability that two randomly chosen elements of $G$ commute (see $[6]$ ).

Therefore,

$$
d(G)=\frac{|\{(x, y) \in G \times G: x y=y x\}|}{|G|^{2}} .
$$

In the year 2001, Lescot [7] has computed $d\left(D_{2 n}\right)$ and $d\left(Q_{2^{n+1}}\right)$ where $D_{2 n}$ is a dihedral group

presented by $\left\langle a, b: a^{n}=b^{2}=1, b a b^{-1}=a^{-1}\right\rangle$ and $Q_{2^{n+1}}$ is a quaternion group presented by $\left\langle a, b: a^{2^{n}}=1, b^{2}=a^{2^{n-1}}, b a b^{-1}=a^{-1}\right\rangle$. It was shown that

$$
d\left(D_{2 n}\right) \rightarrow \frac{1}{4} \quad \text { and } \quad d\left(Q_{2^{n+1}}\right) \rightarrow \frac{1}{4}
$$

as $\left|D_{2 n}\right| \rightarrow \infty$ and $\left|Q_{2^{n+1}}\right| \rightarrow \infty$.

Then Lescot asked, "whether there are other natural families of groups with the same property".

Let $G_{n}$ be a family of finite non-abelian groups such that $\left|G_{n}\right| \rightarrow \infty$ as $n \rightarrow \infty$. Then the limit of $d\left(G_{n}\right)$ as $n \rightarrow \infty$ is called the asymptotic commutativity degree of $G_{n}$. In this regard, the problem posed by Lescot can be restate in the following way:

Question 1: Is there any family of finite groups other than $D_{2 n}$ and $Q_{2^{n+1}}$ whose asymptotic commutativity degree is $\frac{1}{4}$ ?

In the year 2008, Doostie and Maghasedi [2] have computed the commutativity degree of the following classes of finite groups:

$$
\begin{gathered}
G_{1}(m, n)=\left\langle a, b, c: a^{2}=b^{n}=c^{2 m}=1, c^{-1} a c a=1, c^{-1} b c b=1\right\rangle \quad \text { and } \\
G_{2}(m, n)=\left\langle a, b, c: a^{2^{n}}=b^{2^{n}}=c^{2}=1, c^{-1} a c=b, c^{-1} b c=a\right\rangle .
\end{gathered}
$$

They have shown that

$$
d\left(G_{1}(m, n)\right)=\left\{\begin{array}{ll}
\frac{n+3}{4 n}, & \text { if } n \text { is odd } \\
\frac{n+6}{4 n}, & \text { if } n \text { is even }
\end{array} \text { and } d\left(G_{2}(m, n)\right)=\frac{2^{n}+3}{2^{n+2}} .\right.
$$

Therefore, as $n$ tends to infinity we have

$$
d\left(G_{1}(m, n)\right) \rightarrow \frac{1}{4} \text { and } d\left(G_{2}(m, n)\right) \rightarrow \frac{1}{4} .
$$


Thus the families $G_{1}(m, n)$ and $G_{2}(m, n)$ give affirmative answer to Question 1.

In the year 2010, Castelaz (see [1, Chapter 4]) computed the asymptotic commutativity degree for several different classes of finite groups including the dicyclic groups $Q_{4 m}$ presented by $\left\langle a, b: a^{2 m}=1, b^{2}=a^{m}, b a b^{-1}=\right.$ $\left.a^{-1}\right\rangle$ and the semidihedral groups $S D_{n}$ presented by $\left\langle a, b: a^{2^{n-1}}=b^{2}=\right.$ $\left.1, b a b^{-1}=a^{-1+2^{n-2}}\right\rangle$. Castelaz showed that

$$
d\left(Q_{4 m}\right) \rightarrow \frac{1}{4} \text { and } d\left(S D_{n}\right) \rightarrow \frac{1}{4},
$$

as the orders of $Q_{4 m}$ and $S D_{n}$ tend to infinity.

In 2013, the author has computed $d\left(C_{n \theta} C_{2 m}\right)$, where $\theta: C_{2 m} \longrightarrow$ $\operatorname{Aut}\left(C_{n}\right)$ is the homomorphism such that $\theta(b)$, for a generator $b$ of $C_{2 m}$, is the inversing automorphism of $C_{n}$ (see [9]). The author also have shown that

$$
d\left(C_{n} \times{ }_{\theta} C_{2 m}\right) \rightarrow \frac{1}{4} \text { as } n \rightarrow \infty .
$$

Recently, Dutta [3] have shown that the asymptotic commutativity degree of the group $M_{2 m, n}$ for $n>2$ presented by $\left\langle a, b: a^{n}=b^{2 m}=1, b a b^{-1}=\right.$ $\left.a^{-1}\right\rangle$ is $\frac{1}{4}$.

It is worth mentioning that Erovenko and Sury [4] have computed $d(A$ ) $B$ ) where $A, B$ are two finite abelian group and 2 stands for wreath product. They showed that $d(A \prec B) \rightarrow \frac{1}{n^{2}}$ as $|A| \rightarrow \infty$ if $B$ is fixed of order $n>1$.

Doostie and Maghasedi [2] have also computed the commutativity degree of the groups namely $G_{3}(m, n)$ presented by

$$
\left\langle a, b, c: a^{2^{n-1}}=c^{2 m}=1, b^{2}=a^{2^{n-2}}, b^{-1} a b a=c^{-1} a c a=c^{-1} b c b=1\right\rangle .
$$

They have shown that

$$
d\left(G_{3}(m, n)\right)=\frac{2^{n-3}+3}{2^{n}} \text { and so } d\left(G_{3}(m, n)\right) \rightarrow \frac{1}{8}
$$

as $n$ tends to infinity. Motivated by these facts one may ask the following question.

Question 2: Let $k>1$ be any positive integer. Is there any family of finite groups whose asymptotic commutativity degree is $\frac{1}{k}$ ?

In this paper, we answer Question 2 affirmatively. Further we shall show that the reciprocal of every positive integer can be realized as $d(G)$ for some finite group $G$. It is worth mentioning that the central problem in the study of commutativity degree of finite groups is to find the rational numbers in the interval $(0,1]$ that can be realized as $d(G)$ for some finite group $G$. 


\section{Main Results}

We begin with the following three useful results.

Lemma 1. [5] For any two finite group $H$ and $K$ we have

$$
d(H \times K)=d(H) d(K) .
$$

Proposition 2. [10] If $G$ is a finite p-group, where $p$ is a prime, and $G^{\prime} \subseteq$ $Z(G)$, then

$$
d(G)=\frac{1}{\left|G^{\prime}\right|}\left(1+\sum_{\substack{K \leq G^{\prime}, G^{\prime} / K \text { cyclic }}} \frac{(p-1)\left|G^{\prime}: K\right|}{p\left|G: K^{*}\right|}\right)
$$

where $K^{*}=\{x \in G:[G, x] \subseteq K\} \unlhd G$ and $\frac{G}{K^{*}} \cong \prod\left(C_{p^{n_{i}}} \times C_{p^{n_{i}}}\right)$ with $p \leq p^{n_{i}} \leq p^{n_{1}}=p^{k}=\left|G^{\prime}: K\right|$.

A consequence of the above results is given below.

Corollary 3. Let $G$ be a finite group and $\left|G^{\prime}\right|=p$, a prime. If $G^{\prime} \subseteq Z(G)$, then $\frac{G}{Z(G)} \cong\left(C_{p} \times C_{p}\right)^{s}$, for some $s \geq 1$, and

$$
d(G)=\frac{1}{p}\left(1+\frac{p-1}{p^{2 s}}\right) .
$$

Proof. If $G^{\prime} \subseteq Z(G)$ then $G$ is nilpotent of class 2. Hence, $G=$ $P_{1} \times P_{2} \times \cdots \times P_{k}$ where $P_{i}$ 's are Sylow $p_{i}$-subgroups of $G$ corresponding to the primes $p_{i}$ dividing $|G|$. Since $G^{\prime}=P_{1}^{\prime} \times P_{2}^{\prime} \times \cdots \times P_{k}^{\prime}$ and $\left|G^{\prime}\right|=p$ we must have $\left|P_{1}^{\prime}\right|=p$ and $\left|P_{2}^{\prime}\right|=\cdots=\left|P_{k}^{\prime}\right|=1$, assuming that $P_{1}$ is a Sylow $p$-subgroup. Therefore, $P_{2}, \ldots, P_{k}$ are abelian groups and hence $Z(G)=Z\left(P_{1}\right) \times P_{2} \times \cdots \times P_{k}$. By [10, Proposition 2], it follows that

$$
\frac{G}{Z(G)} \cong \frac{P_{1}}{Z\left(P_{1}\right)} \cong\left(C_{p} \times C_{p}\right)^{s}
$$

for some $s \geq 1$. Again, by Lemma 1 and Proposition 2, we have

$$
d(G)=\prod_{i=1}^{m} d\left(P_{i}\right)=d\left(P_{1}\right)=\frac{1}{p}\left(1+\frac{(p-1)\left|P_{1}^{\prime}:\{1\}\right|}{p\left|P_{1}:\{1\}^{*}\right|}\right)
$$


since $K=\{1\}$ is the only proper subgroup of $P_{1}^{\prime}$ such that $P_{1}^{\prime} / K$ is cyclic. Hence, the result follows from $(2.1)$ and $(2.2)$ noting that $\{1\}^{*}=Z\left(P_{1}\right)$.

We now state and prove the first main result of this section which give affirmative answer to Question 2.

Theorem 4. There exists a family of finite groups having asymptotic commutativity degree $\frac{1}{k}$ for every integer $k>1$.

Proof. Let $k=p_{1}^{k_{1}} p_{2}^{k_{2}} \cdots p_{m}^{k_{m}}$ be the prime factorization of $k$. Consider the families $E S\left(n_{i}, p_{i}\right)$ of extra-special $p_{i}$-groups of order $p_{i}^{2 n_{i}+1}$ for $i=$ $1,2, \ldots, m$. By Lemma 1 and Corollary 3 , we have

$$
d\left(\left(E S\left(n_{i}, p_{i}\right)\right)^{k_{i}}\right)=\left(\frac{1}{p_{i}}+\frac{p_{i}-1}{p_{i}^{2 n_{i}+1}}\right)^{k_{i}}
$$

where $\left(E S\left(n_{i}, p_{i}\right)\right)^{k_{i}}$ is the direct product of $k_{i}$ copies of $E S\left(n_{i}, p_{i}\right)$.

Hence, the result follows from Lemma 1, considering the family

$$
\left(E S\left(n_{1}, p_{1}\right)\right)^{k_{1}} \times\left(E S\left(n_{2}, p_{2}\right)\right)^{k_{2}} \times \cdots \times\left(E S\left(n_{m}, p_{m}\right)\right)^{k_{m}}
$$

obtained by extra-special $p$-groups noting that

$$
d\left(\left(E S\left(n_{i}, p_{i}\right)\right)^{k_{i}}\right) \rightarrow \frac{1}{p_{i}^{k_{i}}} \quad \text { as } n_{i} \rightarrow \infty
$$

The following theorem shows that the reciprocal of every positive integer can be realized as $d(G)$ of some finite group $G$.

Theorem 5. There exists a finite group $G$ such that $d(G)=\frac{1}{n}$ for every positive integer $n$.

Proof. We shall prove the theorem by induction on $n$. For $n=1$, we may take $G$ to be any abelian group. If $n=2$, we may take, $G=S_{3}$. So, assume that $n \geq 3$ and that the theorem is true for all positive integers $k$ less than $n$.

Case $1 . \quad n \equiv 0$ or $2(\bmod 4)$. In this case, $n=2^{\alpha} \cdot m$, where $\alpha, m$ are positive integers and $m$ is odd. Clearly $m<n$. So, by induction hypothesis 
there exists a finite group $G$ such that $d(G)=\frac{1}{m}$. Hence, using the fact that $d\left(S_{3}\right)=\frac{1}{2}$ and Lemma 1 , we have

$$
d\left(G \times\left(S_{3}\right)^{\alpha}\right)=d(G) \cdot\left(d\left(S_{3}\right)\right)^{\alpha}=\frac{1}{m \cdot 2^{\alpha}}=\frac{1}{n} .
$$

Case $2 . \quad n \equiv 1 \quad(\bmod 4)$. In this case, $\frac{n+3}{4}$ is a positive integer and $\frac{n+3}{4}<n$. So, by induction hypothesis, there exists a finite group $G$ such that $d(G)=\frac{4}{n+3}$. Hence,

$$
d\left(D_{2 n} \times G\right)=\frac{n+3}{4 n} \cdot \frac{4}{n+3}=\frac{1}{n} .
$$

Case $3 . \quad n \equiv 3 \quad(\bmod 4)$. In this case, $\frac{n+1}{4}$ is a positive integer and $\frac{n+1}{4}<n$. So, by induction hypothesis, there exists a finite group $G$ such that $d(G)=\frac{4}{n+1}$. Hence,

$$
d\left(D_{6 n} \times G\right)=\frac{3 n+3}{12 n} \cdot \frac{4}{n+1}=\frac{1}{n} .
$$

This completes the proof.

We conclude this paper noting that the above two theorems are also obtained by Castelaz considering different families of finite groups (see Corollary 4.3.2 and Corollary 5.3.3 of [1]).

\section{Acknowledgements}

This paper is a part of the author's Ph. D. thesis [8]. The author would like to thank Prof. A. K. Das for his supervision. The author is also thankful to the referee for his/her valuable comments and suggestions which improve the quality of this article.

\section{References}

[1] A. Castelaz, "Commutativity degree of finite groups", M. A. thesis, Wake Forest University, 2010. [On line]. Available: https://bitly/2N9Vca7

[2] H. Doostie and M. Maghasedi, "Certain classes of groups with commutativity degree $\mathrm{d}(\mathrm{G})<\frac{1}{2}$ ", Ars combinatoria, vol. 89, pp. 263-270, Oct. 2008. 
[3] J. Dutta, "On a problem posed by Belcastro and Sherman", Kyungpook mathematical journal, vol. 56, no. 1, pp. 121-123, 2016. [On line]. Available: https://bit.ly/2VXyp5b

[4] I. Erovenko and B. Sury, "Commutativity degree of wreath products of finite abelian groups", Bulletin of the australian mathematical society, vol. 77, no. 1, pp. 31-36, Feb. 2008, doi: $10.1017 / \mathrm{S} 0004972708000038$

[5] W. Gustafson, "What is the probability that two group elements commute?", The American mathematical monthly, vol. 80, no. 9, pp. 1031-1034, Nov. 1973, doi: 10.2307/2318778.

[6] P. Lescot, "Isoclinism classes and commutativity degrees of finite groups", Journal of algebra, vol. 177, no. 3, pp. 847-869, Nov. 1995, doi: $10.1006 /$ jabr.1995.1331.

[7] P. Lescot, "Central extensions and commutativity degree", Сотmunication in algebra, vol. 29, no. 10, pp. 4451-4460, Mar. 2001, doi: 10.1081/AGB-100106768

[8] R. Nath, "Commutativity degree, its generalizations, and classification of finite groups", Ph. D. thesis, North-Eastern Hill University, 2010.

[9] R. Nath, "Computing the commutativity degree of a class of finite groups and consequences", Bulletin of the australian mathematical society, vol. 88, no. 3, pp. 448-452, Dec. 2013, doi: 10.1017/S0004972712001086.

[10] D. Rusin, "What is the probability that two elements of a finite group commute?", Pacific journal of mathematics, vol. 82, no. 1, pp. 237-247, May 1979, doi: 10.2140/pjm.1979.82.237 